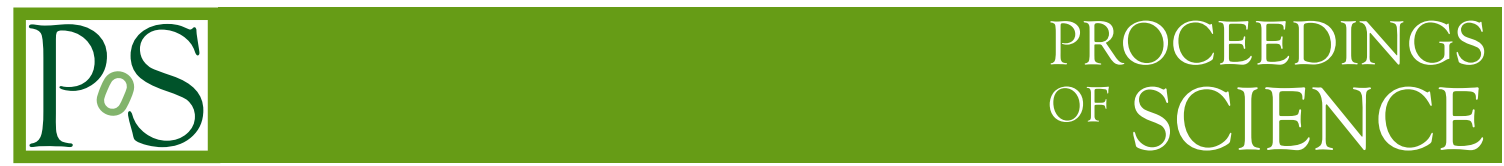

\title{
Numerical calculation of massive multi-loop integrals
}

\section{S. Borowka, G. Heinrich*}

Max-Planck-Institute for Physics, Munich, Germany

E-mail: \{sborowka, gudrun $\}$ @mpp.mpg . de

The program package SECDEC is presented, allowing the numerical evaluation of multi-loop integrals. The restriction to Euclidean kinematics of version 1.0 has been lifted: thresholds can be handled by an automated deformation of the integration contour into the complex plane. Other new features of the program, which go beyond the standard decomposition of loop integrals, are also described. The program is publicly available at http://secdec.hepforge.org.

Loops and Legs in Quantum Field Theory, 11th DESY Workshop on Elementary Particle Physics April 15-20, 2012

Wernigerode, Germany

\footnotetext{
* Speaker.
} 


\section{Introduction}

These days the amount and the precision of high energy collider physics data is increasing rapidly, and the properties of a new discovery have to be studied as detailed as possible. Therefore precise theory predictions are of major importance. While for many observables next-to-leadingorder (NLO) precision is sufficient, there is a considerable number of examples where corrections beyond NLO are required. In these proceedings we present the program SECDEC [1, 2], which can assist the numerical calculation of such corrections in a process independent way, providing examples for multi-loop integrals as well as dimensionally regularised phase space integrals. The program is based on the method of sector decomposition [3-5], which is an algorithm to factorise the poles in the regularisation parameter $\varepsilon$ from complicated multi-parameter integrals. Other public implementations of sector decomposition can be found in [6-9].

While the program SECDEC-1.0 [1] and the public programs mentioned above are limited to kinematics where the values of the Mandelstam invariants and masses have to be such that the denominator of the integrand is guaranteed to be of definite sign, this restriction is lifted in SECDEC2.0 [2]. Integrable singularities located on the real axis are avoided by an automated deformation of the integration contour into the complex plane. The method of contour deformation in a multidimensional parameter space has been pioneered in [10] and later has been refined and applied to various calculations at one loop [11 - 17] and at two loops [18-21].

Numerical methods using dispersion relations, numerical extrapolation, differential equations and/or numerical integration of Mellin-Barnes representations also have been worked out, see e.g. [2228]. However, most of these methods or programs are limited either to specific classes of integrals, or the parameters tuning the numerical integration have to be adapted carefully by the authors in an iterative procedure.

For the program SECDEC-2, the aim was to offer a package which is "multi-purpose", i.e. which can handle very different types of multi-loop/multi-scale integrals in a uniform setup. This universality may come at the expense of sub-optimal performance as compared to dedicated programs for specific classes of diagrams, but on the other hand offers a tool of very wide applicability.

\section{Main features of the program SECDEC}

The program performs the following task: it turns a parameter integral, which can contain ultraviolet and/or infrared singularities regulated by a parameter $\varepsilon$, into a Laurent series in $\varepsilon$, where the coefficients are calculated numerically. For multi-loop integrals, integrable singularities (e.g. due to kinematic thresholds) can also be handled. To start with, the program turns the user information about a Feynman graph (i.e. number of legs, loops, propagators, vertices, on-shell conditions) automatically into the corresponding Feynman parameter representation. The extraction of the poles in $1 / \varepsilon$ is purely algebraic. The coefficients of the poles are sets of finite parameter integrals, which are integrated numerically.

\subsection{General form of multi-loop integrals}

Here we will focus on applications of SECDEC-2 to multi-loop integrals, for details about more general parameter integrals we refer to [1,2]. After Feynman parametrisation, a scalar Feynman 
integral $G$ in $D$ dimensions at $L$ loops with $N$ propagators, where the propagators can have arbitrary, not necessarily integer powers $v_{j}$, can be written as

$$
G=\frac{(-1)^{N_{v}}}{\prod_{j=1}^{N} \Gamma\left(v_{j}\right)} \Gamma\left(N_{v}-L D / 2\right) \int_{0}^{\infty} \prod_{j=1}^{N} d x_{j} x_{j}^{v_{j}-1} \delta\left(1-\sum_{l=1}^{N} x_{l}\right) \frac{\mathscr{U}(\vec{x})^{N_{v}-(L+1) D / 2}}{\mathscr{F}(\vec{x})^{N_{v}-L D / 2}}
$$

with $N_{v}=\sum_{j=1}^{N} v_{j}$. The functions $\mathscr{U}$ and $\mathscr{F}$ can be constructed either directly from the momentum representation or from the topology of the corresponding Feynman graph [29, 30]. The implementation of the construction based on the topology only, without the need to specify the propagators in terms of loop momenta, is one of the new features of SECDEC-2. $\mathscr{U}$ is a positive semi-definite function, whose vanishing is related to the UV subdivergences of the graph. In the region where all Lorentz invariants formed from external momenta are negative, which we will call the Euclidean region, $\mathscr{F}$ is also a positive semi-definite function of the Feynman parameters $x_{j}$ and the invariants. If some of the invariants are zero, for example if some of the external momenta are light-like, the vanishing of $\mathscr{F}$ may induce an IR divergence. Thus it depends on the kinematics and not only on the topology (like in the UV case) whether a zero of $\mathscr{F}$ leads to a divergence or not. Therefore general theorems about the IR singularity structure of multi-loop integrals are sparse, but for practical purposes sector decomposition can provide information about the singularity structure and numerical results, because it offers a constructive algorithm to extract the poles in $1 / \varepsilon$.

\subsection{Deformation of the integration contour}

As mentioned already, the integrand in eq. (2.1) can diverge for certain values of kinematical invariants and Feynman parameters. In cases where this corresponds to an integrable singularity of logarithmic or square root type, related to normal thresholds, we can make use of Cauchy's theorem to avoid the poles on the real axis by a deformation of the integration contour into the complex plane. As long as the deformation is in accordance with the causal $i \delta$ prescription of the Feynman propagators, and no poles are crossed while changing the integration path, the integration contour can be altered such that the convergence of the numerical integration is assured. The $i \delta$ prescription tells us that the contour deformation should be such that the imaginary part of $\mathscr{F}$ is always negative. For real masses and Mandelstam invariants $s_{i j}$, the following ansatz [10, 12] is therefore convenient:

$$
\vec{z}(\vec{x})=\vec{x}-i \vec{\tau}(\vec{x}), \tau_{k}=\lambda x_{k}\left(1-x_{k}\right) \frac{\partial \mathscr{F}(\vec{x})}{\partial x_{k}} .
$$

Unless we are faced with a leading Landau singularity where both $\mathscr{F}$ and its derivatives with respect to $x_{i}$ vanish, the deformation leads to a well behaved integral at the points where the function $\mathscr{F}$ vanishes. In terms of the new variables, we thus obtain

$$
\mathscr{F}(\vec{z}(\vec{x}))=\mathscr{F}(\vec{x})-i \lambda \sum_{j} x_{j}\left(1-x_{j}\right)\left(\frac{\partial \mathscr{F}}{\partial x_{j}}\right)^{2}+\mathscr{O}\left(\lambda^{2}\right)
$$

such that $\mathscr{F}$ acquires a negative imaginary part of order $\lambda$. The size of $\lambda$ determines the scale of the deformation. The initial value for the parameter $\lambda$ can be given by the user; however, the program tries to optimise the value for $\lambda$ for each subsector function and may overwrite the user's choice if the latter is found to be inconvenient. 


\subsection{Installation and usage of the program}

The program can be downloaded from http:// secdec.hepforge.org. Unpacking the tar archive via tar xzvf SecDec.tar.gz will create a directory called SecDec. Running ./install in the SecDec directory will install the package. Prerequisites are Mathematica, version 6 or above, Perl (installed by default on most Unix/Linux systems), a Fortran compiler, or a C++ compiler if the $\mathrm{C}++$ option is used. If contour deformation is required, the $\mathrm{C}++$ option must be used. The libraries CUBA [31, 32] and BASES [33] which are used for the numerical integration come with the package SECDEC.

\section{Usage}

1. Change to the subdirectory loop or general, depending on whether you would like to calculate a loop integral or a more general parameter integral.

2. Copy the files param.input and template.m to create your own parameter and template files myparamfile.input, mytemplatefile.m.

3. Set the desired parameters in myparamfile.input and specify the Feynman graph or the function to evaluate in mytemplatefile.m.

4. Execute the command ./launch - p myparamfile.input -t mytemplatefile.m in the shell.

If you omit the option - p myparamfile.input, the file param. input will be taken as default. Likewise, if you omit the option -t mytemplatefile.m, the file template.m will be taken as default. If your files myparamfile.input, mytemplatefile.m are in a different directory, say, myworkingdir, use the option -d myworkingdir, i.e. the full command then looks like./launch - $d$ myworkingdir - p myparamfile.input -t mytemplatefile.m, executed from the directory SecDec/loop or SecDec/general.

5. Collect the results. If the calculations are done sequentially on a single machine, the results will be collected automatically. If the jobs have been submitted to a cluster, when all jobs have finished, use the command ./results.pl [-d myworkingdir - p myparamfile]. In both cases, the files containing the final results will be located in the graph subdirectory specified in the input file.

\section{New features of the program}

Version 2 of SECDEC contains the following new features.

1. The most important new feature is the fact that multi-scale loop integrals can now be evaluated without restricting the kinematics to the Euclidean region.

2. For scalar multi-loop integrals, the integrand can be constructed from the topology of the diagram, so the user only has to provide the vertices and the propagator masses, but does not have to provide the momentum flow anymore.

3. The files for the numerical integration of multi-scale loop diagrams with contour deformation are written in $\mathrm{C}++$ rather than Fortran. 
4. A parallelisation of the algebraic part for Mathematica versions 7 and higher is possible if several cores are available. Parallelisation of the numerical part is also possible.

5. The so-called primary sector decomposition [3] to eliminate the constraint $\delta\left(1-\sum_{i} x_{i}\right)$ on the Feynman parameters, see eq. (2.1), can be skipped using the -n option, i.e. .launch $-p$ param.input -t template. $m-n$. In this case the program will not assume that such a constraint is present, but immediately proceed to the iterated sector decomposition. This option can be very useful for cases where the user already has done some modifications to the integrand, for example variable transformations which are convenient for a specific integral at hand, or where one or several Feynman parameters already have been integrated out analytically, or for other non-standard integrals. The implementation of this option is very recent and extends the applicability of the program to a much wider class of integrals.

6. The possibility to loop over ranges of numerical values for the Mandelstam invariants, masses (loop case) or user-defined parameters (general case) is automated.

7. To evaluate parametric functions in the subdirectory general, the user can define additional (finite) functions at a symbolic level and specify them only later, after the integrand has been transformed into a set of finite coefficient integrals for each order in $\varepsilon$.

For examples and results, we refer the reader to [2] and the demos directories coming with the program.

\section{Conclusions}

We have presented SECDEC version 2, an automated program which can be applied to multiloop integrals and more general parameter integrals to perform two tasks: factorise dimensionally regulated singularities as poles in $1 / \varepsilon$ and numerically calculate the coefficients of the resulting Laurent series in $\varepsilon$. The program is publicly available at http://secdec.hepforge.org.

An important new feature of the program is the fact that it now can deal with fully physical kinematics, i.e. is not restricted to one-scale problems or Euclidean kinematics anymore. A new construction of the integrand, based entirely on topological rules, is also included, along with other very useful new features which extend the range of applicability of the program.

To calculate full two-loop amplitudes involving several mass scales, the timings still leave room for improvement. However, the program offers the possibility of major parallelisation if several processors are available. An interface to programs performing the reduction to master integrals (not necessarily scalar integrals), which then are fed directly into SECDEC, is under construction.

\section{References}

[1] Carter J and Heinrich G 2011 Comput.Phys.Commun. 182 1566-1581 (Preprint 1011.5493 ).

[2] Borowka S, Carter J and Heinrich G 2012 Comput. Phys. Commun. (Preprint 1204.4152).

[3] Binoth T and Heinrich G 2000 Nucl. Phys. B585 741-759 (Preprint hep-ph/ 0004013 ). 
[4] Roth M and Denner A 1996 Nucl. Phys. B479 495-514 (Preprint hep-ph / 9605420).

[5] Hepp K 1966 Commun. Math. Phys. 2 301-326.

[6] Bogner C and Weinzierl S 2008 Comput. Phys. Commun. 178 596-610 (Preprint 0709 . 4092 ).

[7] Smirnov A and Tentyukov M 2009 Comput.Phys.Commun. 180 735-746 (Preprint 0807 . 4129).

[8] Smirnov A, Smirnov V and Tentyukov M 2011 Comput.Phys.Commun. 182 790-803 (Preprint $0912.0158)$.

[9] Gluza J, Kajda K, Riemann T and Yundin V 2011 Eur.Phys.J. C71 1516 (Preprint 1010.1667 ).

[10] Soper D E 2000 Phys. Rev. 062014009 (Preprint hep-ph/9910292).

[11] Binoth T, Heinrich G and Kauer N 2003 Nucl. Phys. B654 277-300 (Preprint hep-ph/0210 023 ).

[12] Binoth T, Guillet J P, Heinrich G, Pilon E and Schubert C 2005 JHEP 10015 (Preprint hep-ph/0504267).

[13] Nagy Z and Soper D E 2006 Phys. Rev. 074093006 (Preprint hep-ph/ 0610028 ).

[14] Gong W, Nagy Z and Soper D E 2009 Phys. Rev. D79 033005 (Preprint 0812.3686 ).

[15] Lazopoulos A, Melnikov K and Petriello F 2007 Phys. Rev. D76 014001 (Preprint hep-ph/0703273).

[16] Becker S, Reuschle C and Weinzierl S 2010 JHEP 1012013 (Preprint 1010.4187 ).

[17] Becker S, Goetz D, Reuschle C, Schwan C and Weinzierl S 2012 Phys.Rev.Lett. 108032005 (Preprint $1111.1733)$.

[18] Kurihara Y and Kaneko T 2006 Comput.Phys.Commun. 174 530-539 (Preprint hep-ph/0503003).

[19] Anastasiou C, Beerli S and Daleo A 2007 JHEP 05071 (Preprint hep-ph/0 703282 ).

[20] Anastasiou C, Beerli S and Daleo A 2008 Phys. Rev. Lett. 100241806 (Preprint 0803.3065 ).

[21] Freitas A 2012 JHEP 1207132 (Preprint 1205.3515).

[22] Bauberger S, Berends F A, Bohm M and Buza M 1995 Nucl.Phys. B434 383-407 (Preprint hep-ph/9409388).

[23] Caffo M, Czyz H and Remiddi E 2002 Nucl.Phys. B634 309-325 (Preprint hep-ph/ 0203256 ).

[24] Pozzorini S and Remiddi E 2006 Comput.Phys.Commun. 175 381-387 (Preprint hep-ph/0505041).

[25] Czakon M 2006 Comput.Phys.Commun. 175 559-571 (Preprint hep-ph/0511200).

[26] Czakon M 2008 Phys.Lett. B664 307-314 (Preprint 0803.1400 ).

[27] Freitas A and Huang Y C 2010 JHEP 1004074 (Preprint 1001.3243 ).

[28] Yuasa F, de Doncker E, Hamaguchi N, Ishikawa T, Kato K et al. 2012 Comput.Phys.Commun. 183 2136-2144 (Preprint 1112.0637).

[29] Smirnov V A 2006 Feynman integral calculus (Springer).

[30] Heinrich G 2008 Int. J. Mod. Phys. A23 1457-1486 (Preprint 0803.4177 ).

[31] Hahn T 2005 Comput. Phys. Commun. 168 78-95 (Preprint hep-ph/ 0404043 ).

[32] Agrawal S, Hahn T and Mirabella E 2011 (Preprint 1112.0124).

[33] Kawabata S 1995 Comp. Phys. Commun. 88 309-326. 